\title{
CONNECTED DOMINATION CRITICAL GRAPHS WITH CUT VERTICES
}

\author{
PAWATON KAEMAWICHANURAT \\ Theoretical and Computational Science Center \\ Science Laboratory Building and Department of Mathematics \\ Faculty of Science, King Mongkut's University of \\ Technology Thonburi, 126 Pracha Uthit Road, Bang Mod \\ Thung Khru, Bangkok 10140, Thailand \\ e-mail: pawaton.kae@kmutt.ac.th \\ AND \\ NAWARAT ANANCHUEN \\ Center of Excellence in Mathematics \\ CHE, Si Ayutthaya Rd., Bangkok 10400, Thailand \\ e-mail: nawarat.ana@mahidol.ac.th
}

\begin{abstract}
A graph $G$ is said to be $k-\gamma_{c}$-critical if the connected domination number of $G, \gamma_{c}(G)$, is $k$ and $\gamma_{c}(G+u v)<k$ for any pair of non-adjacent vertices $u$ and $v$ of $G$. Let $G$ be a $k-\gamma_{c}$-critical graph and $\zeta(G)$ the number of cut vertices of $G$. It was proved, in $[1,6]$, that, for $3 \leq k \leq 4$, every $k-\gamma_{c}$-critical graph satisfies $\zeta(G) \leq k-2$. In this paper, we generalize that every $k-\gamma_{c^{-}}$ critical graph satisfies $\zeta(G) \leq k-2$ for all $k \geq 5$. We also characterize all $k$ - $\gamma_{c}$-critical graphs when $\zeta(G)$ is achieving the upper bound.
\end{abstract}

Keywords: connected domination, critical.

2010 Mathematics Subject Classification: 05C69.

\section{REFERENCES}

[1] N. Ananchuen, On domination critical graphs with cut vertices having connected domination number 3, Int. Math. Forum 2 (2007) 3041-3052.

doi:10.12988/imf.2007.07277 
[2] G. Chatrand and O.R. Oellermann, Applied and Algorithmic Graph Theory (McGraw-Hill. Inc., New York, 1993).

[3] X.G. Chen, L. Sun and D.X. Ma, Connected domination critical graphs, Appl. Math. Lett. 17 (2004) 503-507. doi:10.1016/S0893-9659(04)90118-8

[4] J.H. Hattingh, N.J. Rad, S.H. Jafari, M. Loizeaux, J. Matthews and L.C. van der Merwe, Total edge critical graphs with leaves, Discrete Math. 312 (2012) $3482-3488$.

doi:10.1016/j.disc.2012.09.001

[5] P. Kaemawichanurat, Connected Domination Critical Graphs, Ph.D. Thesis (Curtin University, 2016).

[6] P. Kaemawichanurat and N. Ananchuen, On 4- $\gamma_{c}$-critical graphs with cut vertices, Util. Math. 82 (2010) 253-268.

[7] P. Kaemawichanurat, L. Caccetta and N. Ananchuen, Critical graphs with respect to total domination and connected domination, Australas. J. Combin. 65 (2016) 1-13.

[8] J.D. Taylor and L.C. van der Merwe, A note on connected domination critical graphs, J. Combin. Math. Combin. Comput. 100 (2017) 3-8.

Received 12 December 2017

Revised 26 June 2018

Accepted 26 June 2018 